\title{
Article
}

\section{Medication-related osteonecrosis of the jaw - a current review}

Domah, Farya, Shah, N., Domah, J. and Shah, Raunaq Pravinchandra

Available at https://clok.uclan.ac.uk/21421/

Domah, Farya, Shah, N., Domah, J. and Shah, Raunaq Pravinchandra (2018) Medication-related osteonecrosis of the jaw - a current review. Oral Surgery, 11 (3). pp. 250-258. ISSN 1752-2471

It is advisable to refer to the publisher's version if you intend to cite from the work. http://dx.doi.org/10.1111/ors.12329

For more information about UCLan's research in this area go to

http://www.uclan.ac.uk/researchgroups/ and search for < name of research Group>.

For information about Research generally at UCLan please go to http://www.uclan.ac.uk/research/

All outputs in CLoK are protected by Intellectual Property Rights law, including Copyright law. Copyright, IPR and Moral Rights for the works on this site are retained by the individual authors and/or other copyright owners. Terms and conditions for use of this material are defined in the policies page.

\section{CLoK}

Central Lancashire online Knowledge www.clok.uclan.ac.uk

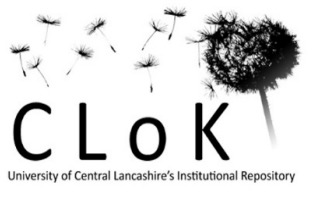




\section{Title: Medication Related Osteonecrosis of the Jaw- a current review}

Keywords: MRONJ, osteonecrosis, jaw, oral, dental

Authors: 1) Dr Farya Domah.

MSc student. Oral surgery.

University of Central Lancashire.

Preston. UK.

2) Dr Nirmal Shah.

MSc student. Aesthetic Medicine.

Queen Mary University of London.

London. UK.

3) Javed Domah

Medical Student.

Trinity College of Dublin.

Dublin. Ireland.

2) Dr Raunaq Shah.

MSc student. Prosthodontics.

University of Central Lancashire.

Preston. UK.

Corresponding author: Dr Farya Domah.

Email address- faryaa@hotmail.com

Number- 07810198697

Date of submission: 30/01/2017 


\section{$\underline{\text { Abstract }}$}

Aim- To discuss different classes of antiresorptive agents and their modes of action. To review the proposed mechanisms in the pathophysiology of osteonecrosis development. To analyse the prevention and the management of medication related osteonecrosis of the jaw.

Materials and methods- Three databases were searched for relevant articles: PubMed, Ovid and Dentistry and Oral Sciences Source. Current literature consists mainly of case series, case reports and expert body opinions.

Discussion- The repertoire of antiresorptive medication keeps expanding as novel drugs are being introduced. The exact pathway that leads to the development of osteonecrosis remains to be elucidated but different mechanisms have been put forward. An attempt at staging has been made and treatment guidelines have been drawn but opinions remain divided. New treatments are being trialled, however, evidence is lacking.

\section{$\underline{\text { Clinical relevance }}$}

Medication related osteonecrosis of the jaw represents a dilemma for the practitioner. It is important for operators to understand the mechanism behind the lesion, prevention and recognition of the lesion and be up to date with the appropriate treatment. As patients at risk of necrosis are on the rise, dentists will be at the forefront of its' prevention. 


\section{Definition}

Osteonecrosis is the death of bone cells due to lack of blood supply (1). The nomenclature 'medication related osteonecrosis of the jaw' (MRONJ) was adopted in 2014 and preferred to the former term 'bisphosphonate related osteonecrosis of the jaw' (BRONJ). This change was to accommodate the new classes of drugs involved in osteonecrosis. These drugs include new angiogenic and antiresorptive agents (2). Table 1 includes a list of antiresorptive medications.

A diagnosis of MRONJ can be made if patients are on current or past treatment with angiogenic or antiresorptive medications, have no history of head and neck malignancy or radiotherapy to the area and have exposed bone for more than eight weeks $(2,3)$.

\section{Incidence}

There is difficulty in obtaining exact incidence data for MRONJ because of underreporting of cases together with discrepancies in case recognition. The available data is extrapolated from retrospective studies with small sample sizes. The incidence in cancer patients on intravenous therapy is between 1.2 to $19 \%$. For patients being treated by oral medication for osteoporosis, the risk of MRONJ is 1 in 1262 per year. In the UK, there are on average 620 new cases a year (4-6).

Table 1: Antiresorptive medications and indications (7) 


\section{$\underline{\text { Antiresorptive medication }}$}

\section{Bisphosphonates}

The majority of MRONJ cases are from bisphosphonates. Bisphosphonates are a group of antiresorptive medication effective on bone. Non-nitrogen containing bisphosphonates like etidronate have been approved by the Food and Drug Administration (FDA) since 1977 and have not been involved in ONJ. Nitrogen containing bisphosphonates ( $\mathrm{N}$-bisphosphonates) like pamidronate have been circulated since 1991 and are reported to be up to 700 times more potent than etidronate. Pamidronate, because of its' higher potency, does not get metabolised. It is the accumulation of the drug over time that predisposes patients to osteonecrosis. Bisphosphonates are thought to work by inhibition of osteoclastic activity $(8,9)$. Other proposed mechanisms include alteration of bone turnover and inhibition of angiogenesis $(2,3,8)$. The mode of action is discussed in further detail later in the text.

\section{Receptor activator of nuclear factor kappa B ligand (RANKL) inhibitor}

Denusomab is a fully human monoclonal antibody and a potent antiresorptive agent. RANKL is a member of the tumour necrosis factor (TNF) superfamily and is the main mediator of osteoclastic bone resorption (7). RANKL binds to its receptor RANK to produce and activate osteoclasts and suppress their apoptosis. Denusomab inhibits binding of RANKL to its receptor, reducing number of functioning osteoclasts, achieving near total suppression of osteoclastogenesis (10). Denusomab

reduces the risk of bone fracture by increasing bone density (7). Denusomab affects production of osteoclasts whereas bisphosphonates affect their resorptive power. However, their efficacies as antiresoptive agents are the same (10).

\section{$\underline{\text { Anabolic agents }}$}

Recombinant human parathyroid hormone, teriparatide, encourages bone formation through osteoblast activation. It is believed that teriparatide may emerge as the medication of choice for osteoporosis. Early studies have shown resolution of osteonecrosis with teriparatide treatment (7). 


\section{$\underline{\text { Antiangiogenic agents }}$}

Vascular endothelial growth factor (VEGF) inhibitor.

Bevacizumab is a recombinant human monoclonal antibody that inhibits angiogenesis by inhibiting VEGF. It is used in combination with standard chemotherapy for treatment of metastatic cancer (11).

\section{Tyrosine kinase receptor (TKR) inhibitor}

TKR inhibitors like sunitinib are used for treatment of renal carcinoma and gastrointestinal tumours. Sunitinib inhibits cellular signalling by targeting multiple TKRs. Among these multiple TKRs, are receptors responsible for tumour cell proliferation and VEGF responsible for angiogenesis (11).

\section{Pathophysiology}

Although MRONJ was recognised more than 10 years ago, its' pathophysiology is not fully understood. With the emergence of ONJ caused by new medications like denusomab, researchers are focusing on mechanisms common to both bisphosphonates and denusomab. The common hypotheses are inhibition of osteoclastic activity and remodelling, bone turnover, infection and inflammation, inhibition of angiogenesis, genetic predisposition, immune dysfunction and idiopathic $(2,3,8)$.

\section{Inhibition of osteoclastic activity and remodelling}

$\mathrm{N}$-bisphosphonates pass into the cell and inhibit prenylation of guanosine triphosphate proteinase; which are required for survival of working osteoclasts (12). Non-N-bisphosphonates also enter the cell by endocytosis. The agent then accumulates in the cell up to a toxic level (13).

Osteoblasts differentiate into osteocytes. Secretion of hydroxyapatite crystals in the collagen matrix of mineralised bone then surrounds the osteocytes.

Bone formation follows the release of cytokines such as bone morphogenic protein (BMP) and insulin like growth factors 1 and 2. The cytokines induce mesenchymal cells and osteoprogenitors to differentiate into osteoblasts. Osteoclasis causes the release of the aforementioned cytokines. As the bisphosphonates affect osteoclasts, normal osteoclasis is affected, which in turn affects the remodel- 
ling. When the surrounded osteocytes exceed their life span of 150 days, they cannot maintain the structural integrity of the matrix and microfractures develop (9).

It is postulated that, like bisphosphonates, denusomab also leads to an end effect of decreased osteoclastic activity and remodelling by inhibiting osteoclasts and increasing apoptosis. Bone remodelling occurs via the same mechanism throughout the whole skeleton. However, osteonecrosis has been observed primarily in the alveolar process of the bi-maxilla. Animal studies have shown reduced bone remodelling of the jaws. This supports the idea that necrosis occurs preferentially in the jaws because of increased bone turnover at the site. Other antiresorptive medications like denusomab have been found to cause similar rates of osteonecrosis as bisphosphonates. This fact further strengthens the pivotal part of decreased bone remodelling in ONJ (2). Bisphosphonates can be taken up by any endocytic cells; especially osteoclasts. Osteoclasts are preferred because they reside in Howship's lacunae and release carbonic anhydrase to dissolute bone. The acid easily releases bisphosphonates from the matrix. High concentrations of the drug in Howship's lacunae and surroundings are believed to affect proximate osteoblasts, but evidence is lacking.

Haematopoietic cells and adipocytes of the bone marrow can also take up bisphosphonates. Accumulation of the drug can affect function which is demonstrated by the fevers, myalgias and arthralgias experienced by users after administration of high dose intravenous bisphosphonates. In vitro studies have explained the role of osteoblasts, fibroblasts and oral keratinocytes in necrosis. Their cell life and multiplication are negatively affected by bisphosphonates in a dose dependent relationship. This new information provides understanding of the way other cells are affected by bisphosphonates but it is not possible to measure the individual drug concentrations in patients' tissues. While the effect of bisphosphonates on bone marrow cells are acknowledged, the occurrence of necrosis in denusomab patients suggest these effects are not critical in causing osteonecrosis. These observations reiterate the major role of decreased bone remodelling in causing ONJ $(13,14)$.

\section{Bone turnover}

It has been proposed that low bone turnover; the main feature of successful treatment with antiresorptive drugs, plays a role in osteonecrosis. The increased risk of osteonecrosis with higher doses 
of antiresorptive drugs backs up this theory $(3,10)$. However, considerably reduced osteoclasis caused by the drugs would result in osteopetrosis and not osteonecrosis. Osteonecrosis is not associated with other conditions of low bone turnover like hypoparathyroidism. High osteoclast numbers have been detected in bone samples of lesion sites, whereby bone lysis is presented radiographically. Canine studies demonstrated areas of spontaneous bone necrosis with dead osteocytes in subjects treated with high dose antiresorptives (3). Several researchers found no suppression in bone turnover in patients with osteonecrosis $(3,15,16)$. Nuclear bone scanning of patients with metastatic breast carcinoma treated with antiresorptives failed to demonstrate high bone turnover in the maxilla and mandible. Developing microfractures following low bone turnover may precipitate osteonecrosis, however supporting evidence is lacking (10)

\section{Inflammation and infection}

The first reported cases of osteonecrosis were following dental extractions; which pointed to surgical trauma as the cause. Dental extractions are usually due to pathology. Several studies have implicated inflammation and infection in the development of osteonecrosis. It is however indeterminate as to whether necrosis comes before or after infection. It has been demonstrated that antiresorptive medication coupled with inflammation or infection alone are enough to prompt osteonecrosis. Infection could explain local osteolysis normally found in osteonecrotic sites despite patients being on high dose antiresorptive medication (10). Certain bacterial by-products can increase local cytokine production and other microbes can have direct effects on B cells and receptor activator nuclear factor kB ligand (RANKL), culminating in increased osteoclastic action (17).

Histology of excised necrotic jaw specimens identified bacteria, viruses and fungi; co-existing in biofilms (2). Actinomyces may be an important factor in high grade osteonecrosis and is isolated in most osteonecrosis specimens (18). Presence of actinomyces at osteonecrotic sites may be opportunistic because oral actinomyces are common but actinomycosis is uncommon (19). The polysaccharide matrix that makes up the biofilm often conceals presence of pathogens to microscopy (10). Polymorphonuclear leukocytes have also been found in biofilms. This cluster of microorganisms may directly contribute to ONJ. Khan et al. proposed that bisphosphonates hinder cell division and viability of oral epithelium. This hindrance together with dental infection or trauma is thought to further 
damage the structure of oral mucosa, leading to necrosis (3). However, an in-vitro study did not demonstrate any effect on cell migration by alendronate or zoledronate. They concluded that suppression of oral keratinocytes is not a factor in osteonecrosis. (20).

The risk of developing ONJ from inflammation, infection and following surgical procedures is increased in patients on intravenous bisphosphonates (16). Via a strict preventative dental programme, Bramati et al. reduced the occurrence of ONJ in oncology patients. This highlights the role of bacterial infection in osteonecrosis (21). In animals, researchers have induced osteonecrosis from bacterial infection alone (2). They have also prevented the onset of osteonecrosis with antibiotics and mucoperiosteal flap coverage following exodontia (10). The acidic environment created by infection releases bisphosphonates from their matrix. High concentrations of bisphosphonates affect osteoblasts and facilitate osteonecrosis development.

Oral infection attracts macrophages to the area (22). Bisphosphonates, following their administration, are in circulation shortly before they become bound to bone. During this period of circulation, macrophages can be ahead of osteoclasts to phagocytose bisphosphonates (23). With repeated intake of bisphosphonates, active bone sites decrease, which allows non-bone cells to take up the drug. Macrophages in contact with bisphosphonates lose their ability to defend against micro-organism invasion (15).

\section{Inhibition of angiogenesis}

Angiogenesis is the process of forming new blood vessels. Angiogenic growth factors like VEGF activate receptors on endothelial cells which proliferate, forming new vessels. Angiogenesis is fundamental in cancer metastasis through tumour invasion of vessels. Osteonecrosis was historically termed avascular necrosis of the jaw and angiogenesis was the prime hypothesis in osteonecrosis histopathology. N-bisphosphonates are known to inhibit angiogenesis. Laboratory experiments have reliably shown downregulation of angiogenesis with zoledronate (2). In oncology patients on intravenous zoledronate, VEGF and platelet derived growth factors were reduced (24). Osteonecrosis of other bones is occurring in patients with new antiangiogenic drugs like TKR inhibitors and monoclonal antibody targeting VEGF $(2,3)$. Denusomab related cases of osteonecrosis have been reported, however, denusomab is not known to inhibit angiogenesis. Moreover, biopsy sample analysis of os- 
teonecrosis lesions found vasculature to be patent in most cases. Osteonecrosis appears in the maxilla as well as the mandible (10). However, the mandible can be up to 9 times more affected than the maxilla (25). While the maxilla is highly vascularised, the dense bone of the mandible coupled with its' comparatively lower vascularity could explain the difference in occurrence rate (25). Vascular volume in animals was unchanged after administration of N-bisphosphonates (3). Due to the lack of hard evidence linking angiogenesis to osteonecrosis, the term 'avascular necrosis' was dropped.

\section{Immunosuppression}

Immunosuppression has been proposed as a risk for osteonecrosis. Chemotherapy drugs cause immunosuppression by either suppressing the bone marrow function or from the high dose of glucocorticoid (26). Sonis et al. could only consistently induce osteonecrosis in rats when zolendronic acid was combined with dexamethasone (27). Dexamethasone causes delayed wound healing and alteration in microflora, predisposing the subject to infection (10). Further investigation on bisphosphonates and dexamethasone showed that $\mathrm{T}$ regulatory cells 17 , which participate to control and prevent autoimmune disease, were activated. At the same time, regulatory $\mathrm{T}$ cells which modulate the immune system were suppressed. Mesenchymal stem cells reversed the osteonecrosis, thereby strengthening the theory of immune dysfunction as a cause for osteonecrosis (28). Bisphosphonates act as gamma delta T cells receptor agonists and increase production of proinflammatory cytokines followed by diminution of T cells, impairing immune function (3).

Vitamin D influences the immune system and is vital for calcium absorption. Animals with vitamin D deficiency treated with bisphosphonates, developed osteonecrosis following exodontia. Thus, immunodeficiency could predispose oncology patients to osteonecrosis (26).

\section{Genetic predisposition}

In patients with similar comorbidities receiving same drug treatment, the development of osteonecrosis is not standardised. Genetics may play a role in its' development. The suggestions are alterations in farnesyl pyrophosphate synthase or cytochrome P450-CYP2C8 genes. Genetic studies are ongoing in this area (3). 


\section{Cytotoxicity}

Bisphosphonate toxicity can be to hard or soft tissues. Toxicity could be from long term use, high doses and accumulation of bisphosphonates in bone tissue. Bisphosphonates act locally and not systemically, mainly affecting the jaws (4). Necrotic areas with dead osteocytes were found in animals treated with bisphosphonates. The toxic effects of the bisphosphonates caused the cell death but suppression of bone remodelling could also be a plausible explanation for the necrotic areas. $\mathrm{N}$ bisphosphonates inhibit cell division by inhibition of farnesyl diphosphate synthase found in osteoclats (10).

Although bisphosphonates bind to bone, soft tissue toxicity has been described. Several cell types including cervical and oral epithelial cells have exhibited decreased cell multiplication after contact with bisphosphonates in vitro. In vivo, patients have experienced gastrointestinal symptoms after administration of bisphosphonates. As bisphosphonates are excreted via the kidneys a few hours after administration, the window for uptake by non-bone cells is small (2). Experiments showed the doses of bisphosphonates required to halt cell proliferation was similar to those found in patients receiving intravenous zoledronate. In previously exposed bone, dental surgery may result in bisphosphonate release into the area. High drug concentrations can prevent replication of oral epithelial cells, lengthening mucosal healing (10). Denusomab is not known to cause cytotoxicity.

\section{Local factors}

Local trauma from surgical procedures like exodontia, endodontic or periodontal surgery is a trigger factor MRONJ development. A study found that $60 \%$ of osteonecrosis cases were caused by dentoalveolar insult/trauma. Spontaneous osteonecrosis in absence of antiresorptives has been reported after endotracheal intubation. This emphasises the role played by alveolar bone trauma in the development of osteonecrosis (4).

Figure1. Proposed mechanisms for the development of ONJ (10) 
Figure 2. Proposed mechanisms for ONJ development (4)

\section{Management}

\section{Staging}

A staging system for osteonecrosis allows data collection on an international level, directing treatment strategies and assessing prognosis of patients on antiresorptive medication. The system includes the stages '0' to ' 3 '. The International Task Force on Osteonecrosis of the Jaw in 2015 was of the opinion that 'Stage 0' may lead to overestimation of the disease's prevalence. It argues that wrong diagnoses of osteonecrosis may be made in absence of clinical evidence of necrotic bone (3). However, the American Association of Oral and Maxillofacial Surgeons (AAOMS) has defended the use of 'Stage 0' as numerous studies have described up to $50 \%$ of stage 0 disease progressing to higher stages (2). 


\section{Prevention}

Several studies have found a lesser occurrence of osteonecrosis in patient groups where preventative measures were applied via a multidisciplinary unit. This would involve patients undergoing a comprehensive dental check-up before commencing antiresorptive therapy. Any source of acute infection or potential infection in the oral cavity would be identified and rectified to prevent exacerbation once the drug therapy has begun (2). Smaller studies have shown that good quality endodontic treatment with adequate coronal seal as opposed to extractions could reduce the risk of MRONJ $(29,30)$.

\section{Cessation of therapy before oral surgery}

There is no consensus on a 'drug holiday' i.e. discontinuation of the drug for a definite period. It has been recommended that patients on antiresorptives for less than two years need not stop their medication, while patients on the drugs for more than four years would stop the medication until the surgical site healed. In 2011, the FDA stated that there was not enough evidence available to reach a consensus.

Free bisphosphonate radicals are in circulation for a short time before excretion by the kidneys. The rest is bound to osteoclasts, which have a life span of two weeks. The serum level of bisphosphonates after two months would be very low, making this an adequate period before oral surgery (2). Some authors have suggested a three-month drug holiday for patients on denusomab (31). Since the terminal half-life of bisphosphonates is about 10 years, the usefulness of a drug holiday is questioned (32). The evidence base to support a drug holiday is weak and is mainly drawn from expert opinions.

\section{Patients with established osteonecrosis}

With established osteonecrosis, the aim of treatment is to keep patients free of pain and infection. There is also emphasis on maintaining the dentition to avoid requiring future dental surgery. In less advanced stages, conservative treatment is advised. In stages 3 or in cases where sequestrums are present, resection is favoured. Smoothening of rough necrotic areas will remove the source of irritation and allow optimal soft tissue healing (2). 
Opposing the AAOMS, Ristow supported surgical intervention in disease stages lower than ' 3 ', quoting success rates of $85 \%$. Conservative treatment is regarded by some as palliative treatment whereas surgical treatment is regarded as curative treatment. The main surgical approaches are debridement and resection. Debridement involves curettage to reduce necrotic bone. Resection is the removal of all the necrosis up to the margin of vital bone. Tension free mucoperiosteal coverage of the site offers the best healing chance. As necrotic bone does not revascularise, it should be thoroughly removed. Small remnants may cause recurrence or progression of the osteonecrosis. Also, irregular surfaces of the exposed bone are conducive to microbial colonisation; which is involved in the pathogenesis of osteonecrosis $(31,33)$.

Non-removal of exposed bone, it can be argued, will put patients at risk of future infection, especially in the case of immunocompromised patients. However, conservative treatment is useful in patients undergoing chemotherapy or whose medical statuses' do not allow surgical intervention. Even though these measures are seen as temporary ones, $70 \%$ of patients remain pain free $(31,34)$.

\section{Emerging treatments}

Growth factors like VEGF induce angiogenesis and osteoblastogenesis at injury site, promoting soft tissue healing. Growth factors, applied topically after resection, have achieved success in refractory osteonecrosis cases. Surgical lasers have been used for bony resections. Ozone therapy has increased haemoglobin concentration and number of red blood cells in avascular necrosis. Hyperbaric oxygen has several cellular benefits including wound healing through promotion of angiogenesis at wound peripheries, collagen formation and decrease of leukocyte adhesion (35). Mucosal healing following hyperbaric oxygen therapy has indeed been investigated. Although it showed improvement in the pain and quality of life scores, there was no statistical difference with the control group. Teriparatide, a powerful stimulant of both osteoclastic and osteoblastic activity is showing promising results. Reversal of inhibitory effects of bisphosphonates have been observed in vivo.

Tocopherol and pentoxyfilline used in conjunction with tetracyclines have alleviated symptoms in patients and decreased areas of exposed bone $(2,37,38)$. Pentoxyfilline increases oxygen delivery to 
tissues by increasing blood flow, decreasing blood viscosity and increasing cellular flexibility (36). Tocopherol improves endothelial function by its' potent scavenging action on free oxygen radicals which impact necrosis (36). There is not yet enough evidence available to support any of these treatment modalities $(2,37,38)$.

\section{$\underline{\text { Conclusion }}$}

Antiresorptives and antiangiogenic drugs are indispensable in the treatment of malignant and skeletal conditions. As more data is collected on these novel drugs, pathophysiology will be better understood. From there, more robust clinical guidelines, backed up by solid evidence can be formulated. To reach that stage, it is imperative that dentists, maxillofacial surgeons and oncologists work as a team and communicate effectively.

\section{References}

(1) Dorland WAN. In: Saunders, editor. Dorland's Illustrated Medical Dictionary. Vol 31st ed ed. Philadelphia: Elsevier; 2007. p. 1368.

(2) Ruggiero SL, Dodson TB, Fantasia J, Goodday R, Aghaloo T, Mehrotra B, et al. American Association of Oral and Maxillofacial Surgeons position paper on medication-related osteonecrosis of the jaw--2014 update. Journal of Oral \& Maxillofacial Surgery 2014 Oct;72(10):1938-1956.

(3) Khan AA, Morrison A, Hanley DA, Felsenberg D, McCauley LK, O'Ryan F, et al. Diagnosis and management of osteonecrosis of the jaw: a systematic review and international consensus. Journal of Bone \& Mineral Research 2015 Jan;30(1):3-23.

(4) Badel T, Pavicin IS, Carek AJ, Rosin-Grget K, Grbesa D. Pathophysiology of osteonecrosis of the jaw in patients treated with bisphosphonate. Coll Antropol 2013 2013;37(2):645-651.

(5) Rogers S, Palmer N, Lowe D, Randall C. United Kingdom nationwide study of avascular necrosis of the jaws including bisphosphonate-related necrosis. British Journal of Oral and Maxillofacial Surgery 2015;53(2):176-182.

(6) Ruggiero SL. Guidelines for the diagnosis of bisphosphonate-related osteonecrosis of the jaw (BRONJ). Clin Cases Miner Bone Metab 2007 Jan;4(1):37-42.

(7) Hellstein JW, Adler RA, Edwards B, Jacobsen PL, Kalmar JR, Koka S, et al. Managing the care of patients receiving antiresorptive therapy for prevention and treatment of osteoporosis: executive summary of recommendations from the American Dental Association Council on Scientific Affairs. J Am Dent Assoc 2011;142(11):1243-1251. 
(8) Bamias A, Kastritis E, Bamia C, Moulopoulos LA, Melakopoulos I, Bozas G, et al. Osteonecrosis of the jaw in cancer after treatment with bisphosphonates: incidence and risk factors. $J$ Clin Oncol 2005 Dec 1;23(34):8580-8587.

(9) Marx RE. Pamidronate (Aredia) and zoledronate (Zometa) induced avascular necrosis of the jaws: a growing epidemic. Journal of Oral and Maxillofacial Surgery 2003;61(9):1115-1117.

(10) Katsarelis H, Shah NP, Dhariwal DK, Pazianas M. Infection and medication-related osteonecrosis of the jaw. J Dent Res 2015 Apr;94(4):534-539.

(11) Troeltzsch M, Woodlock T, Kriegelstein S, Steiner T, Messlinger K, Troeltzsch M. Physiology and pharmacology of nonbisphosphonate drugs implicated in osteonecrosis of the jaw. J Can Dent Assoc 2012;78(c85):1-7.

(12) Dunford JE, Rogers MJ, Ebetino FH, Phipps RJ, Coxon FP. Inhibition of protein prenylation by bisphosphonates causes sustained activation of Rac, Cdc42, and Rho GTPases. Journal of bone and mineral research 2006;21(5):684-694.

(13) Allen MR, Ruggiero SL. A review of pharmaceutical agents and oral bone health: how osteonecrosis of the jaw has affected the field. Int J Oral Maxillofac Implants 2014 Jan-Feb;29(1):e45-57.

(14) Adverse effects of bisphosphonates: implications for osteoporosis management. Mayo Clinic Proceedings: Elsevier; 2009.

(15) Pazianas M. Osteonecrosis of the jaw and the role of macrophages. J Natl Cancer Inst 2011 Feb 2;103(3):232-240.

(16) Reid IR, Cornish J. Epidemiology and pathogenesis of osteonecrosis of the jaw. Nature Reviews Rheumatology 2012;8(2):90-96.

(17) Belibasakis GN, Bostanci N, Hashim A, Johansson A, Aduse-Opoku J, Curtis MA, et al. Regulation of RANKL and OPG gene expression in human gingival fibroblasts and periodontal ligament cells by Porphyromonas gingivalis: a putative role of the Arg-gingipains. Microb Pathog 2007;43(1):46-53.

(18) De Ceulaer J, Tacconelli E, Vandecasteele S. Actinomyces osteomyelitis in bisphosphonaterelated osteonecrosis of the jaw (BRONJ): the missing link? European Journal of Clinical Microbiology \& Infectious Diseases 2014;33(11):1873-1880.

(19) Naik NH, Russo TA. Bisphosphonate-related osteonecrosis of the jaw: the role of actinomyces. Clin Infect Dis 2009 Dec 1;49(11):1729-1732.

(20) McLeod NM, Moutasim KA, Brennan PA, Thomas G, Jenei V. In vitro effect of bisphosphonates on oral keratinocytes and fibroblasts. Journal of Oral and Maxillofacial Surgery 2014;72(3):503-509.

(21) Bramati A, Girelli S, Farina G, Dazzani MC, Torri V, Moretti A, et al. Prospective, monoinstitutional study of the impact of a systematic prevention program on incidence and outcome of osteonecrosis of the jaw in patients treated with bisphosphonates for bone metastases. J Bone Miner Metab 2015;33(1):119-124.

(22) Geissmann F, Manz MG, Jung S, Sieweke MH, Merad M, Ley K. Development of monocytes, macrophages, and dendritic cells. Science 2010 Feb 5;327(5966):656-661.

(23) Coxon FP, Thompson K, Roelofs AJ, Ebetino FH, Rogers MJ. Visualizing mineral binding and uptake of bisphosphonate by osteoclasts and non-resorbing cells. Bone 2008;42(5):848-860. 
(24) Santini D, Vincenzi B, Dicuonzo G, Avvisati G, Massacesi C, Battistoni F, et al. Zoledronic acid induces significant and long-lasting modifications of circulating angiogenic factors in cancer patients. Clin Cancer Res 2003 Aug 1;9(8):2893-2897.

(25) Grisar K, Schol M, Schoenaers J, Dormaar T, Coropciuc R, Vander Poorten V, et al. Osteoradionecrosis and medication-related osteonecrosis of the jaw: similarities and differences. Int J Oral Maxillofac Surg 2016;45(12):1592-1599.

(26) Yamashita J, McCauley LK, Van Poznak C. Updates on osteonecrosis of the jaw. Current Opinion in Supportive and Palliative Care 2010 September 2010;4(3):200-206.

(27) Sonis ST, Watkins BA, Lyng GD, Lerman MA, Anderson KC. Bony changes in the jaws of rats treated with zoledronic acid and dexamethasone before dental extractions mimic bisphosphonaterelated osteonecrosis in cancer patients. Oral Oncol 2009;45(2):164-172.

(28) Kikuiri T, Kim I, Yamaza T, Akiyama K, Zhang Q, Li Y, et al. Cell-based immunotherapy with mesenchymal stem cells cures bisphosphonate-related osteonecrosis of the jaw-like disease in mice. Journal of Bone and Mineral Research 2010;25(7):1668-1679.

(29) Katz H. Endodontic implications of bisphosphonate-associated osteonecrosis of the jaws: a report of three cases. J Endod 2005;31(11):831-834.

(30) Dioguardi M, Troiano G, Caloro G, Cocco A. Endodontic Re-treatment of a Tooth with a Floor Perforation in a Patient in Treatment with Oral Bisphosphonate. J Gen Practice 2016;4(252):2.

(31) Ristow O, Otto S, Troeltzsch M, Hohlweg-Majert B, Pautke C. Treatment perspectives for medication-related osteonecrosis of the jaw (MRONJ). Journal of Cranio-Maxillo-Facial Surgery 2015 Mar;43(2):290-293.

(32) Kimmel D. Mechanism of action, pharmacokinetic and pharmacodynamic profile, and clinical applications of nitrogen-containing bisphosphonates. J Dent Res 2007;86(11):1022-1033.

(33) Fukushima Y, Enoki Y, Nakaoka C, Okubo M, Kokabu S, Nojima J, et al. Usability of surgical treatment in cases of bisphosphonate-related osteonecrosis of the jaw stage 2 with sequestrum. Annals of Maxillofacial Surgery 2015 Jan-Jun;5(1):67-70.

(34) Bodem JP, Kargus S, Engel M, Hoffmann J, Freudlsperger C. Value of nonsurgical therapeutic management of stage I bisphosphonate-related osteonecrosis of the jaw. Journal of Cranio-MaxilloFacial Surgery 2015 Sep;43(7):1139-1143.

(35) Gill A, Bell CN. Hyperbaric oxygen: its uses, mechanisms of action and outcomes. QJM 2004;97(7):385-395.

(36) Epstein MS, Wicknick FW, Epstein JB, Berenson JR, Gorsky M. Management of bisphosphonate-associated osteonecrosis: pentoxifylline and tocopherol in addition to antimicrobial therapy. An initial case series. Oral Surgery, Oral medicine, Oral Pathology, Oral Radiology, and Endodontology 2010;110(5):593-596.

(37) Barba-Recreo P, Del Castillo Pardo de Vera JL, Georgiev-Hristov T, Ruiz Bravo-Burguillos E, Abarrategi A, Burgueno M, et al. Adipose-derived stem cells and platelet-rich plasma for preventive treatment of bisphosphonate-related osteonecrosis of the jaw in a murine model. Journal of CranioMaxillo-Facial Surgery 2015 Sep;43(7):1161-1168. 
(38) Fliefel R, Troltzsch M, Kuhnisch J, Ehrenfeld M, Otto S. Treatment strategies and outcomes of bisphosphonate-related osteonecrosis of the jaw (BRONJ) with characterization of patients: a systematic review. International Journal of Oral \& Maxillofacial Surgery 2015 May;44(5):568-585. 
